\title{
December 2014 Pulmonary Case of the Month: Bronchiolitis in Adults
}

\author{
Kashif Yaqub, MD \\ Lewis J. Wesselius, MD \\ Department of Pulmonary Medicine \\ Mayo Clinic Arizona \\ Scottsdale, AZ
}

\section{History of Present IIIness}

A 61-year-old man was admitted to the hospital with cough, dyspnea and hypoxemia. He had some prior respiratory symptoms about a month prior to admission, but his symptoms worsened recently. He was seen in Family Medicine Clinic on the day of admission and noted to have saturations of $88-$ $89 \%$.

A thoracic CT scan was done shortly after his initial symptoms but was negative for lung consolidation or pulmonary embolus. He currently was having fever with temperatures of 99 to 103 degrees and cough that was only slightly productive.

\section{$\mathrm{PMH}, \mathrm{FH}, \mathrm{SH}$}

He had a history of hypertension and obstructive sleep apnea although he was not using continuous positive airway pressure (CPAP).

\section{Medications}

- nifedipine

- lisinopril/hydrochlorothiazide

- cough medication

\section{Physical Examination}

General: SpO2 95\% on 2l/min by nasal cannula

Chest: his breath sounds were coarse, but there were no wheezes or crackles.

Cardiovascular: regular rate and rhythm with no murmur noted

Extremities: no clubbing, cyanosis or edema.

Skin: no rashes noted.

\section{Laboratory}

CBC: Hemoglobin $15.1 \mathrm{~d} / \mathrm{dL}$, WBC $15.3 \times 10^{3}$ cells/ $\mu \mathrm{L}$, no eosinophilia. Procalcitonin: $0.22 \mathrm{ng} / \mathrm{mL}$ (normal $<0.15 \mathrm{ng} / \mathrm{mL}$ ). Nasopharyngeal swab: PCR negative for pertussis, chlamydophila and mycoplasma pneumonia.

\section{Radiography}

His thoracic CT scan at the initial presentation of his illness about a month prior to admission was reviewed (Figure 1). 

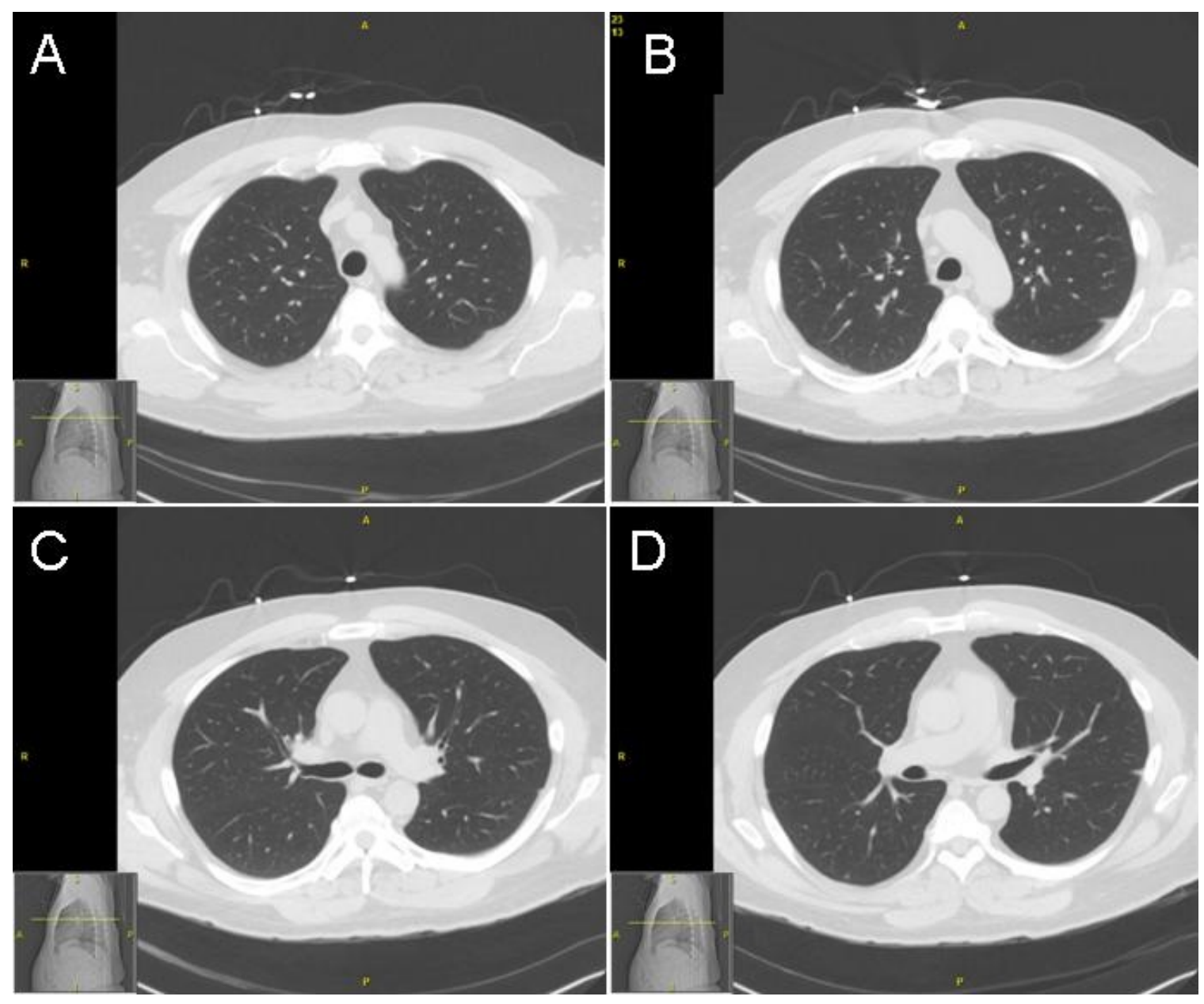

Figure 1. Representative images from thoracic CT scan in lung windows done about one month prior to admission.

Which of the following are appropriate at this time?

1. Cocci serology

2. Empirically begin antibiotics for community-acquired pneumonia

3. Repeat the thoracic CT scan

4. Sputum culture

5. All of the above 


\section{Correct! \\ 5. All of the above}

It is unclear what is the cause of the patient's cough, dyspnea and hypoxemia. Valley fever is certainly possible and a sputum culture with empiric antibiotics is reasonable. The thoracic CT scan from a month prior to admission is normal and a repeat CT scan seems justified (Figure 2).

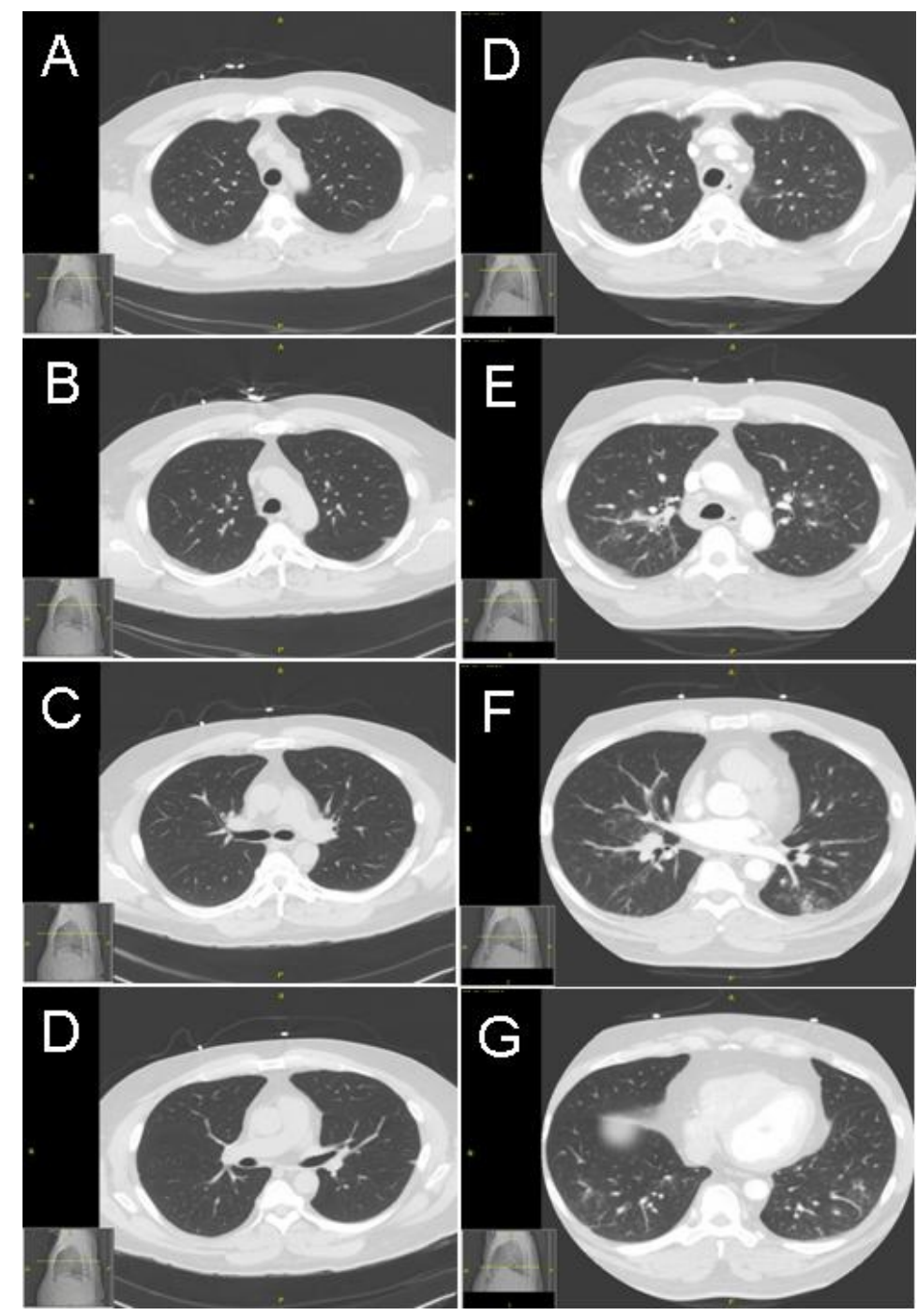

Figure 2. Repeat thoracic CT scan in lung windows.

What is the next most appropriate step?

1. Begin amphotericin

2. Begin fluconazole

3. Bronchoscopy with bronchoalveolar lavage

4. Switch the antibiotics to an aminoglycoside and methicillin

5. Video-assisted thorascopic surgery (VATS) 


\section{Correct! \\ 3. Bronchoscopy with bronchoalveolar lavage}

The repeat CT scan does not show classic pneumonia but a few scattered central nodules and a "tree-in-bud" pattern. Although switching the antibiotics or adding anti-fungals is not wrong, there is no evidence of a fungal infection at this time. VATS seems overly aggressive in a non-immunocompromised host. Therefore, the best choice seems to be bronchoscopy with bronchoalveolar lavage.

Bronchoscopy revealed areas of marked bronchial erythema (Figure 3).

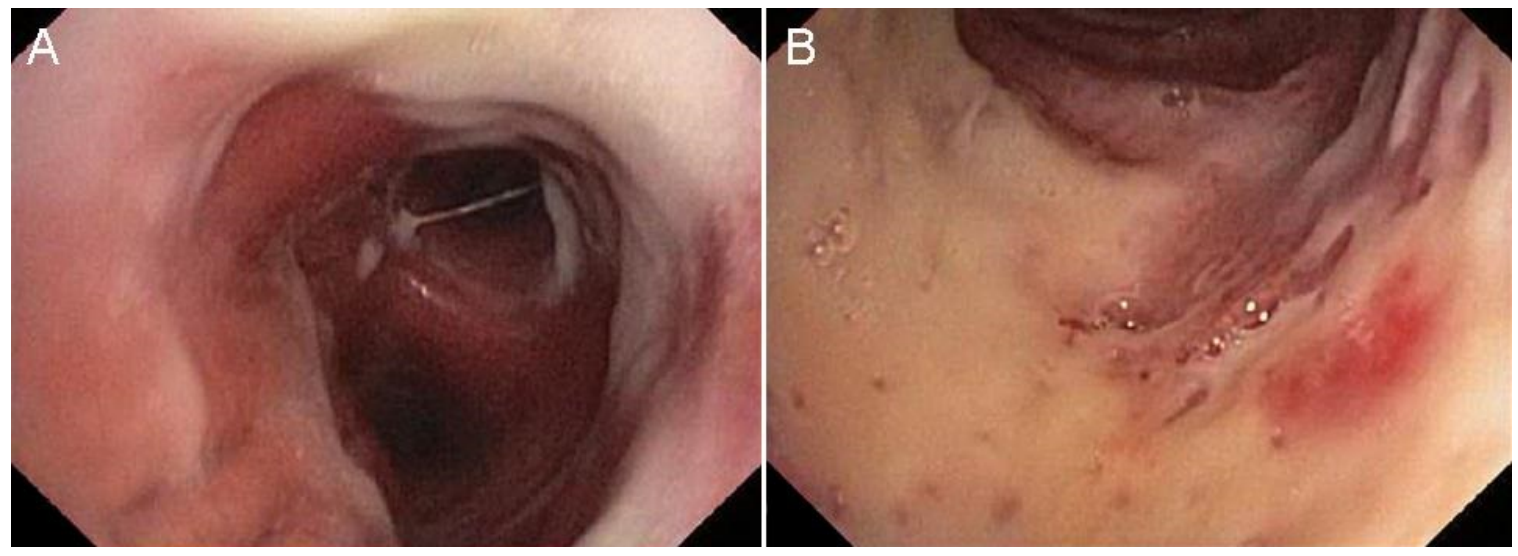

Figure 3. Selected views from the bronchoscopy showing areas of marked bronchial erythema.

A viral respiratory panel performed on the bronchoalveolar lavage fluid was negative. However, bacterial PCR was positive for Mycoplasma.

What is the next appropriate step?

1. Begin corticosteroids

2. Begin macrolide antibiotics

3. Begin penicillin

4. Observe

5. VATS to confirm the diagnosis 


\section{Correct! \\ 2. Begin macrolide antibiotics}

Macrolides (e.g., azithromycin) are generally considered the treatment of choice for mycoplasma in both children and adults (1). In addition to macrolides, fluoroquinolones are recommended for the treatment of adults and tetracyclines (e.g., doxycycline) can be used for older children and adults. All mycoplasmas lack a cell wall and, therefore, all are inherently resistant to beta-lactam antibiotics (e.g., penicillin). Resistance to macrolides has been emerging in Mycoplasma since 2000. Current data suggest that the prevalence of macrolide resistance in M. pneumoniae is probably rising in the United States, possibly due to increased use of azithromycin in recent years to treat a number of illnesses.

The patient does not have classic pneumonia but has bronchiolitis, an inflammatory process of small airways $(<2 \mathrm{~mm})$. Although this is most commonly seen in children secondary to viral infection, adults may also develop broncholitis. Possible causes in adults include inhalational injury, infection, druginduced, connective tissue disease, graft vs. host disease, ulcerative colitis, primary biliary cirrhosis and idiopathic (2). This patient is rather typical in both clinical and radiographic appearance for broncholitis.

Although classically associated with pneumonia, Mycoplasma may also case a broncholitis picture (3). In the series reported by Cha (3), $27 \%$ of cases of Mycoplasma infection showed this pattern. Nasopharnygeal swab PCR has limited sensitivity for Mycoplasma infections (40\%) similar to the results in this patient. Overall the prognosis is good, although some patients with residual restrictive changes have been reported. Our patient responded rapidly to macrolide antibiotic therapy.

\section{References}

1. CDC. Mycoplasma pneumoniae infection. Available at: http://www.cdc.gov/pneumonia/atypical/mycoplasma/hcp/antibiotic-treatmentresistance.html (accessed 11/24/14).

2. Colby TV, Epler GR, Gruden JF. Bronchiolar disorders. In: Crapo JD, ed. Atlas of pulmonary medicine. 4th ed. Philadelphia: Current Medicine Group/Springer, 2008:69-84.

3. Cha SI, Shin KM, Jeon KN, Yoo SS, Lee J, Lee SY, Kim CH, Park JY, Jung $\mathrm{TH}$. Clinical relevance and characteristics of pleural effusion in patients with Mycoplasma pneumoniae pneumonia. Scand J Infect Dis. 2012;44(10):793-7. [CrossRef] [PubMed] 